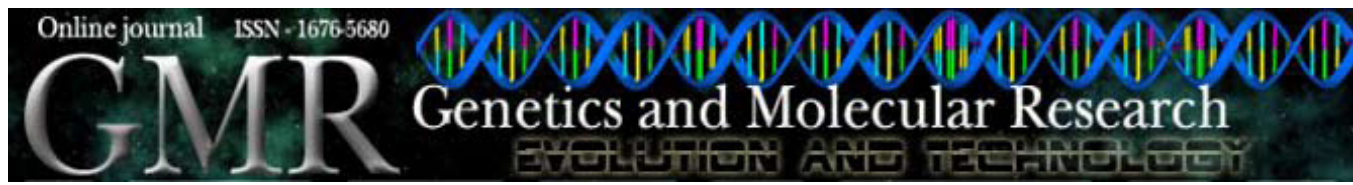

Short Communication

\title{
Application of 16S rRNA, cytochrome b and control region sequences for understanding the phylogenetic relationships in Oryx species
}

\author{
H.A. Khan, I.A. Arif, A.A. Al Homaidan and A.H. Al Farhan \\ Molecular Fingerprinting and Biodiversity Unit, \\ Prince Sultan Research Chair Program for Environment and Wildlife, \\ College of Science, King Saud University, Riyadh, Saudi Arabia \\ Corresponding author: H.A. Khan \\ E-mail: khan_haseeb@yahoo.com
}

Genet. Mol. Res. 7 (4): 1392-1397 (2008)

Received September 29, 2008

Accepted October 9, 2008

Published December 16, 2008

\begin{abstract}
The present study reports the application of mitochondrial markers for the molecular phylogeny of Oryx species, including the Arabian oryx (AO), scimitar-horned oryx ( $\mathrm{SHO}$ ) and plains oryx (PO), using the Addax as an outgroup. Sequences of three molecular markers, 16S rRNA, cytochrome $\mathrm{b}$ and a control region, for the above four taxa were aligned and the topologies of respective phylogenetic trees were compared. All these markers clearly differentiated the genus Addax from Oryx. However, for species-level grouping, while 16S rRNA and cytochrome b produced similar phylogeny (SHO grouped with $\mathrm{PO}$ ), the control region grouped $\mathrm{SHO}$ with $\mathrm{AO}$. Further studies are warranted to generate more sequencing data, apply multiple bioinformatics tools and to include relevant nuclear markers for phylogenetic analysis of Oryx species.
\end{abstract}

Key words: Oryx; Endangered species; Conservation; Mitochondrial DNA; Phylogenetic relationship 


\section{INTRODUCTION}

The oryx antelopes prefer dry, near-desert conditions and can survive without water for days or even weeks. Massive destruction and deterioration of their habitat and illegal hunting have decimated oryx populations significantly, and now these animals are almost extinct in the wild. Since captive breeding is known to play an important role in the conservation of threatened species, several oryx breeds have been successfully retained in captivity after extinction in the wild. Mesochina et al. (2003) have built-up a captive oryx population recognized as the most polymorphic of all captive herds, suggesting that no recent managementrelated bottleneck has occurred. However, genetic analysis has suggested that perhaps as much as half of the neutral genetic variation present in the pre-extinction population of the Arabian oryx is absent from contemporary populations (Marshall et al., 1999). The Arabian oryx, though formerly distributed throughout the Arabian Peninsula, was extirpated from the wild by hunting in the early 1970s (Henderson, 1974) but preserved in captivity and reintroduced in certain protected areas (Ostrowski et al., 1998; Spalton et al., 1999; Mesochina et al., 2003). A global perspective about developing more effective captive breeding programs is necessary to maintain the genetic diversity for saving this endangered species (Iyengar et al., 2007).

The use of molecular approaches can significantly contribute to captive breeding and reintroduction strategies for the conservation of various endangered animals such as the oryx (Russello and Amato, 2007). Mitochondrial DNA (mtDNA) is regarded as an important tool in studying evolutionary relationships among various taxa due to its conserved protein-coding regions, high variability in non-coding sequences and lack of recombination (Olivo et al., 1983; Ingman et al., 2000). The sequence divergence accumulates more rapidly in mtDNA than nuclear DNA due to faster mutation rate and lack of repair system in mtDNA rendering it to often contain high levels of informative variation. Although mtDNA has evolved faster than the nuclear genome, the rate of evolution is different for different regions of mtDNA and has been used to examine various phylogenetic relationships. Since 12S rDNA is highly conserved, it is usually applied to illustrate the phylogeny of higher categorical levels such as phyla or subphyla, whereas $16 \mathrm{~S}$ rDNA is commonly used for phylogenetic studies at midcategorical levels such as families or genera (Gerber et al., 2001). However, the mitochondrial protein-coding genes and the control region (CR) evolve much faster and are therefore considered to be powerful tools for inferring evolutionary history in lower categorical levels such as genera and species. This investigation describes the potential of $16 \mathrm{~S}$ rRNA, cytochrome $b$ and CR sequences for understanding the molecular phylogeny of Oryx species.

\section{MATERIAL AND METHODS}

The sequences of 16S rRNA, cytochrome b and CR of the three Oryx species including the Arabian oryx (Oryx leucoryx), scimitar-horned oryx (Oryx dammah) and plains oryx (Oryx gazella) were obtained from GenBank. The respective sequences of the Addax (Addax nasomaculatus) represented the outgroup due to close relationship of Addax and Oryx yet representing a separate sister taxa (Hassanin and Douzery, 1999; Iyengar et al., 2006). Details of all these sequences including the GeneBank accession numbers and their lengths are summarized in Table 1. The taxonomic classification of Oryx species is as follows: Kingdom (Animalia); Phylum (Chordata); Subphylum (Vertebrata); Class (Mammalia); Order (Artiodactyla); 
Family (Bovidae); Subfamily (Hippotraginae); Genus (Oryx); Species (Oryx leucoryx, Oryx dammah or Oryx gazella). The outgroup Addax differed at the genus level with the following classification: Genus (Addax); Species (Addax nasomaculatus).

\begin{tabular}{|c|c|c|c|}
\hline Taxon & mtDNA region & GenBank accession No. & No. of base pairs \\
\hline \multirow{3}{*}{$\begin{array}{l}\text { Arabian oryx } \\
\text { (Oryx leucoryx) }\end{array}$} & $16 \mathrm{~S}$ rRNA & U87021 & 342 \\
\hline & Cytochrome b & AF036286 & 1143 \\
\hline & Control region & AJ235326 & 1253 \\
\hline \multirow{3}{*}{$\begin{array}{l}\text { Scimitar-horned oryx } \\
\text { (Oryx dammah) }\end{array}$} & $16 \mathrm{~S}$ rRNA & U87020 & 342 \\
\hline & Cytochrome b & AJ222685 & 1143 \\
\hline & Control region & AJ235324 & 1261 \\
\hline \multirow{3}{*}{$\begin{array}{l}\text { Plains oryx } \\
(\text { Oryx gazella) }\end{array}$} & $16 \mathrm{~S}$ rRNA & U87022 & 342 \\
\hline & Cytochrome b & AF249973 & 1140 \\
\hline & Control region & AJ235325 & 1237 \\
\hline \multirow{3}{*}{$\begin{array}{l}\text { Addax } \\
\text { (Addax nasomaculatus) }\end{array}$} & $16 \mathrm{~S}$ rRNA & U87023 & 342 \\
\hline & Cytochrome b & AF034722 & 1143 \\
\hline & Control region & AJ235310 & 1324 \\
\hline
\end{tabular}

The sequences were first examined for alignment using the BioEdit software (Hall, 1999). Phylogenetic analyses were performed using the evolutionary distances computed by the maximum composite likelihood method in MEGA 4 (Tamura et al., 2004, 2007). Estimates of average evolutionary divergence over all sequence pairs were computed by averaging the number of base substitutions per site. The evolutionary history was inferred using the maximum parsimony method (Eck and Dayhoff, 1966). The bootstrap consensus trees inferred from 1000 replicates (Felsenstein, 1985) are taken to represent the evolutionary history of the taxa analyzed.

\section{RESULTS AND DISCUSSION}

The results of sequence alignment showed the absence of any indels in 16S rRNA and cytochrome $\mathrm{b}$ genes, whereas numerous indels were incorporated for a satisfactory alignment of CR sequences. Specific indels in the CR of Oryx species have been reported earlier (Iyengar et al., 2006). Table 2 shows the estimates of evolutionary divergence, which are based on the pairwise analysis of sequences from four taxa. The overall mean distances based on the number of nucleotide substitutions per site were found to be 0.026 , 0.062 and 0.140 for $16 \mathrm{~S}$ rRNA, cytochrome $\mathrm{b}$ and $\mathrm{CR}$, respectively. The phylogenetic relationships of the four taxa, on the basis of $16 \mathrm{~S}$ rRNA, cytochrome $\mathrm{b}$ and CR sequence similarity/diversity, are shown as bootstrap consensus trees (Figure 1). All three segments of mtDNA clearly differentiated Addax from Oryx. However, the topologies of phylogenetic trees resulting from $16 \mathrm{~S}$ rRNA and cytochrome $\mathrm{b}$, although similar to one another, were different from CR-based phylogeny (Figure 1). The scimitar-horned oryx was grouped with the plains oryx using $16 \mathrm{~S}$ rRNA or cytochrome $\mathrm{b}$ but with the Arabian oryx using CR (Figure 1). 
Table 2. Estimates of evolutionary divergence between sequences from the four taxa.

\begin{tabular}{|c|c|c|c|c|c|c|c|c|c|}
\hline & \multicolumn{3}{|c|}{ 16S rRNA } & \multicolumn{3}{|c|}{ Cytochrome b } & \multicolumn{3}{|c|}{ Control region } \\
\hline & $\mathrm{AO}$ & PO & SO & $\mathrm{AO}$ & PO & SO & $\mathrm{AO}$ & PO & SO \\
\hline $\mathrm{AO}$ & - & & & - & & & - & & \\
\hline PO & 0.012 & - & & 0.059 & - & & 0.143 & - & \\
\hline SO & 0.012 & 0.018 & - & 0.051 & 0.046 & - & 0.111 & 0.134 & - \\
\hline $\mathrm{AD}$ & 0.030 & 0.043 & 0.043 & 0.075 & 0.073 & 0.070 & 0.157 & 0.158 & 0.139 \\
\hline
\end{tabular}

$\overline{\mathrm{AO}}=$ Arabian oryx $\mathrm{PO}$ = plains oryx; $\mathrm{SO}=$ scimitar-horned oryx; $\mathrm{AD}=$ Addax.

\section{$16 \mathrm{~S}$ rRNA}

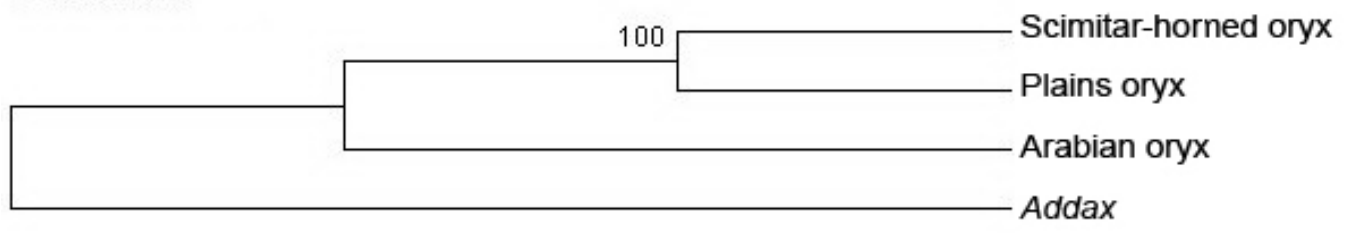

\section{Cytochrome b}

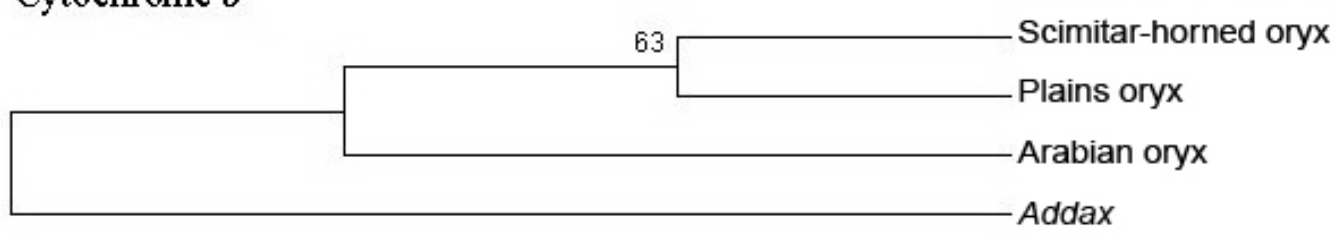

\section{Control region}

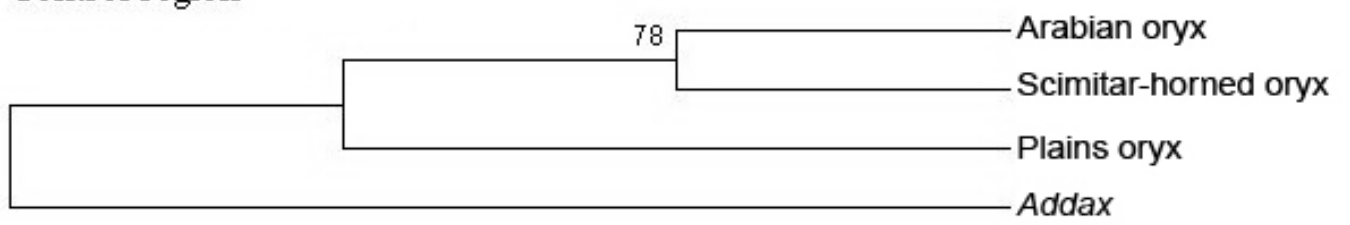

Figure 1. Phylogenic relationships of various Oryx species using Addax as an outgroup. The evolutionary history was inferred using the maximum parsimony method, and the bootstrap consensus trees inferred from 1000 replicates are taken to represent the phylogeny.

The mean evolutionary distances were comparatively greater for CR (5.38-fold) and cytochrome b (2.38-fold) as compared to $16 \mathrm{~S}$ rRNA. Consequently, the faster-evolving CR marker increased the resolution in phylogenetic trees; however, a conflict was noticed between the topologies of trees resulting form $16 \mathrm{~S}$ rRNA or cytochrome $b$ and CR, suggesting the independent nature of these markers (Figure 1). These differential phylogenies may be associated with comparatively high variations in the non-coding $\mathrm{CR}$ region than in the coding region of the cytochrome $\mathrm{b}$ gene due to reduced functional constraints and relaxed selection pressure. 
Although, increased polymorphism in the CR segment may render it superior to cytochrome $b$ for species or sub-species level identification, the possibility of reduced phylogenic information due to back mutations and parallel substitutions in rapidly evolving CR may not be ruled out. It is also important to mention that changing the out-group species or the length of CR segment can significantly alter the topology of phylogenic trees (Iyengar et al., 2006).

Allard et al. (1992) studied bovid phylogeny using the complete $12 \mathrm{~S}$ and 16S rRNA genes and suggested that most bovid tribes originated early in the Miocene with all extant lineages present by approximately 16-17 million years ago. Gatesy et al. (1997) performed a phylogenetic analysis of 26 bovid taxa using $12 \mathrm{~S}$ and $16 \mathrm{~S}$ rRNA sequences and demonstrated antelopes as a paraphyletic assemblage. Moreover, the basal diversification of bovid tribes, which was largely unresolved by morphological, immunodiffusion, allozyme, and protein sequence data, remained unresolved with the use of rRNA-based molecular markers (Gatesy et al., 1992). Matthee and Davis (2001) have shown conservative nuclear DNA data to be highly effective in resolving associations among bovid subfamilies, suggesting that the subfamilies Alcelaphinae (hartebeest, tsessebe, wildebeest) and Hippotraginae (roan, sable, gemsbok) share a close evolutionary relationship and together form a sister clade to the more primitive Caprinae (sheep, goat, muskox).

In conclusion, this preliminary study demonstrates the potential application of mitochondrial markers in understanding the relatedness among various Oryx species. Both cytochrome $\mathrm{b}$ and $\mathrm{CR}$ segments appear to be independent indicators of the phylogenetic relationship among Oryx species. However, further studies are warranted to generate more sequencing data and to utilize multiple bioinformatics and statistical tools to ensure the accuracy and reliability of the phylogenetic analysis of Oryx species.

\section{REFERENCES}

Allard MW, Miyamoto MM, Jarecki L, Kraus F, et al. (1992). DNA systematics and evolution of the artiodactyl family Bovidae. Proc. Natl. Acad. Sci. U. S. A. 89: 3972-3976.

Eck RV and Dayhoff MO (1966). Atlas of Protein Sequence and Structure. National Biomedical Research Foundation, Silver Springs.

Felsenstein J (1985). Confidence limits on phylogenies: an approach using the bootstrap. Evolution 39: 783-791.

Gatesy J, Yelon D, DeSalle R and Vrba ES (1992). Phylogeny of the Bovidae (Artiodactyla, Mammalia), based on mitochondrial ribosomal DNA sequences. Mol. Biol. Evol. 9: 433-446.

Gatesy J, Amato G, Vrba E, Schaller G, et al. (1997). A cladistic analysis of mitochondrial ribosomal DNA from the Bovidae. Mol. Phylogenet. Evol. 7: 303-319.

Gerber AS, Loggins R, Kumar S and Dowling TE (2001). Does nonneutral evolution shape observed patterns of DNA variation in animal mitochondrial genomes? Annu. Rev. Genet. 35: 539-566.

Hall TA (1999). BioEdit: a user-friendly biological sequence alignment editor and analysis program for Windows 95/98/ NT. Nucleic Acids Symp. Ser. 41: 95-98.

Hassanin A and Douzery EJ (1999). The tribal radiation of the family Bovidae (Artiodactyla) and the evolution of the mitochondrial cytochrome b gene. Mol. Phylogenet. Evol. 13: 227-243.

Henderson DS (1974). Were they the last Arabian Oryx? Oryx 12: 347-350.

Ingman M, Kaessmann H, Paabo S and Gyllensten U (2000). Mitochondrial genome variation and the origin of modern humans. Nature 408: 708-713.

Iyengar A, Diniz FM, Gilbert T, Woodfine T, et al. (2006). Structure and evolution of the mitochondrial control region in oryx. Mol. Phylogenet. Evol. 40: 305-314.

Iyengar A, Gilbert T, Woodfine T, Knowles JM, et al. (2007). Remnants of ancient genetic diversity preserved within captive groups of scimitar-horned oryx (Oryx dammah). Mol. Ecol. 16: 2436-2449.

Marshall TC, Sunnucks P, Spalton JA, Greth A, et al. (1999). Use of genetic data for conservation management: the case 
of the Arabian oryx. Anim. Conserv. 2: 269-278.

Matthee CA and Davis SK (2001). Molecular insights into the evolution of the family Bovidae: a nuclear DNA perspective. Mol. Biol. Evol. 18: 1220-1230.

Mesochina P, Bedin E and Ostrowski S (2003). Reintroducing antelopes into arid areas: lessons learnt from the oryx in Saudi Arabia. C. R. Biol. 326 (Suppl 1): S158-S165.

Olivo PD, Van de Walle MJ, Laipis PJ and Hauswirth WW (1983). Nucleotide sequence evidence for rapid genotypic shifts in the bovine mitochondrial DNA D-loop. Nature 306: 400-402.

Ostrowski S, Bedin E, Lenain D and Abuzinada AH (1998). Ten years of Arabian oryx conservation breeding in Saudi Arabia - achievements and regional perspectives. Oryx 32: 209-222.

Russello MA and Amato G (2007). On the horns of a dilemma: molecular approaches refine ex situ conservation in crisis. Mol. Ecol. 16: 2405-2406.

Spalton JA, Lawrence MW and Brend SA (1999). Arabian oryx reintroduction in Oman: successes and setbacks. Oryx 33: $168-175$.

Tamura K, Nei M and Kumar S (2004). Prospects for inferring very large phylogenies by using the neighbor-joining method. Proc. Natl. Acad. Sci. U. S. A. 101: 11030-11035.

Tamura K, Dudley J, Nei M and Kumar S (2007). MEGA4: Molecular Evolutionary Genetics Analysis (MEGA) software version 4.0. Mol. Biol. Evol. 24: 1596-1599. 